\title{
Tagungsbericht
}

Marlies Ockenfeld, Darmstadt

\section{Entscheiden unter Risiko und Unsicherheit}

\author{
SVP-Fachtagung 2015
}

DOI 10.1515/iwp-2015-0050

Mit einem neuen Rekord von knapp 80 Teilnehmerinnen und Teilnehmern hat die Heidelberger SVP Deutschland AG am 16. Juni 2015 in der lichtdurchfluteten Heidelberger Print \& Media Academy ihre 10. Fachtagung Market Intelligence in Zusammenarbeit mit dem dcif (Deutsches Competitive Intelligence Forum) e. V. und dem Bundesverband mittelständische Wirtschaft durchgeführt. Der Erfolg dieser immer wieder anregenden Veranstaltung liegt sicherlich zum großen Teil darin, dass sie Themen aufgreift, die erfahrungsgemäß wenig später wichtig werden und ins allgemeine Bewusstsein gelangen, die interessant sind und die den Veranstaltern selbst Spaß machen. Tim Brouwer, Vorstand der SVP Deutschland AG, machte in seiner Begrüßung die Zielsetzung des Treffens klar: es soll die „Market Intelligencer“ zusammenbringen und ihnen mit den ausgewählten Themen einen Wissensvorsprung verschaffen, den sie gegenüber Vorgesetzten und Kollegen zur Sicherung der eigenen Position ausspielen können. Überall geraten nämlich die Intermediäre unter Druck; auch Ärzte und Juristen bekommen Probleme, weil einfache Auskünfte inzwischen von den Klien-

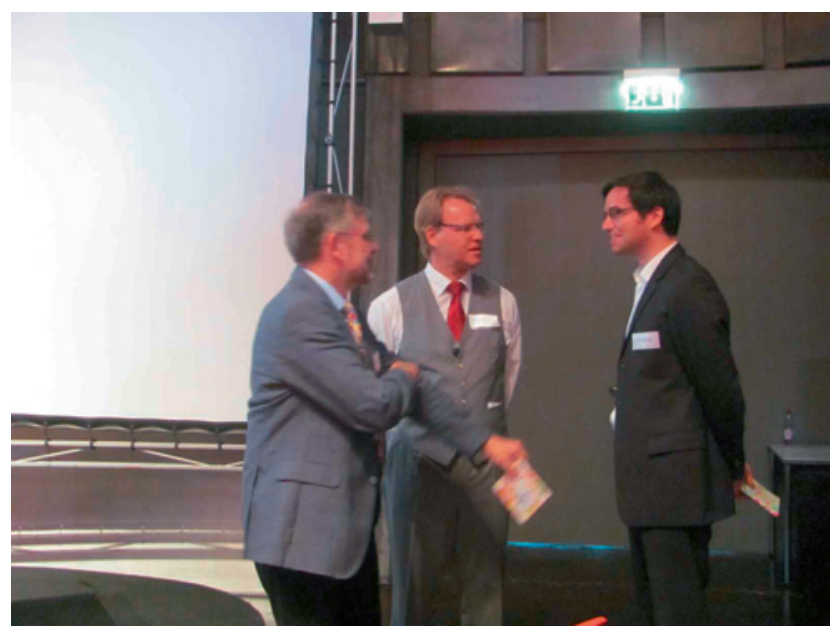

Abbildung 1: Tim Brouwer (Mitte) im Gespräch mit den beiden Vortragsrednern Gunter Dueck und Florian Artinger (Foto: Marlies Ockenfeld). ten über digitale Angebote selbst eingeholt werden. Nur Spezialisten werden überleben, Routineaufgaben werden mehr und mehr automatisiert oder werden in Selbsthilfe organisiert.

Will man Trends erspüren, nützen nur selten Zahlen, man braucht vielmehr eine auf Wissen und Erfahrungen beruhende Intuition. Brouwer erläuterte eingangs noch einmal den Unterschied zwischen Market Research (Marktforschung) und Market Intelligence - eine griffige deutsche Bezeichnung hierfür hat die Branche bisher nicht hervorgebracht. Marktforschung bedient sich in Verbrauchermärkten erfolgreich repräsentativer Umfragemethoden und liefert „Komma-Zahlen“, die häufig nicht hinterfragt werden. Repräsentativität wird auch in anderen Märkten häufig nachgefragt, ist dort aber nicht relevant. In B2B- und in Nischenmärkten ist es sinnvoller mit bruchstückhaften Informationen ein stimmiges Bild der Wirklichkeit zu entwerfen. Die Vorgehensweise ähnelt eher der von Kriminalisten, die aufgrund von Beobachtungen und Indizien Zusammenhänge erkennen und dafür sorgen, dass schließlich eine richtige Entscheidung getroffen werden kann. Marktforschung benötigt viel Zeit, Market Intelligence geht schnell. Eine probate Methode ist es, zu falsifizieren, also zu prüfen, ob es Anzeichen dafür gibt, dass eine Idee mit großer Sicherheit scheitern wird. Solche Knock-out-Kriterien findet man mit MarketIntelligence-Methoden schnell.

Risiken und Wahrscheinlichkeiten werden in Deutschland häufig falsch eingeschätzt. Viele haben nicht ausreichend trainiert, gute Risiken, also solche die die zwar Unsicherheiten bergen, aber einem nicht das Genick brechen können, die Chancen bieten, von schlechten Risiken zu unterscheiden.

\section{Pragmatische Bauch- entscheidungen mit Heuristiken}

Dr. Florian Artinger, Ökonom und Verhaltensforscher vom Max-Planck-Institut für Bildungsforschung, lieferte in seinem programmatischen Vortrag „Risiko - welche 
Strategien funktionieren bei Unsicherheit ... und wie? Heuristische Strategien als pragmatische Konzepte zum Umgang mit der Ungewissheit“ Ergebnisse aus seinen wissenschaftlichen Forschungsprojekten. Zusammen mit zwei Kollegen und dem Institutsleiter Prof. Gerd Gigerenzer arbeitet er auch für das Spin-off Simply Rational, Institut für Entscheidungen des MPI.

Müssen Entscheidungen unter Unsicherheit getroffen werden, so ist eine häufig angewandte Strategie, sich auf Prognosen zu stützen. Leider sind viele Prognosen weit weg von der tatsächlichen Entwicklung. Als Beispiel demonstrierte er die Prognosen von 22 international renommierten Banken hinsichtlich der Entwicklung des Wechselkurses von US\$ und Euro im Zeitraum 2001 bis 2010 und verglich sie mit dem tatsächlichen Verlauf der Kursentwicklung. Dass Prognosen von Wirtschaftsforschungsinstituten und Banken selten richtig liegen, weiß man als Zeitungsleser. Die Frage ist, warum ihnen dennoch immer wieder große Bedeutung zugemessen wird.

Artinger wies auf den Unterschied zwischen Risiko (risk) und Unsicherheit (uncertainty) hin und auf die unterschiedlichen Strategien im Umgang damit. Bei Risiken sind die Eintrittswahrscheinlichkeiten bekannt, man kann sie mit mathematischen Methoden, mit Logik und Statistik berechnen. Bei Unsicherheit helfen selten komplizierte mathematische Ausdrücke, man braucht Heuristiken, um möglichen Entscheidungsoptionen zu finden.

Heuristiken sind einfache Entscheidungsregeln, die in wenigen Sätzen formuliert und bewusst oder unbewusst angewendet werden. Sie brauchen nur ein Minimum an Informationen. Als Beispiel veranschaulichte er das Auffangen eines Balls, ein Vorgang, der sich zwar mit einer komplizierten mathematischen Gleichung beschreiben lässt, doch jeder Ballspieler lernt intuitiv die aus drei Schritten bestehende einfache Faustregel: 1. Fixiere den Ball, 2. Beginne zu laufen, 3. Passe die Laufgeschwindigkeit so an, dass der Blickwinkel konstant bleibt. Artinger bezeichnete diese Regel als Blickwinkelheuristik.

Mit drei Beispielen erläuterte er typische Heuristiken im Bereich Markt und Finanzen. Ein Vergleich der MeanVariance-Methode des Nobelpreisträgers Harry Markowitz mit der einfachen Heuristik „Verteile dein Geld zu gleichen Teilen auf jeden von N Fonds" führte beim Vergleich der beiden Wertpapierdepots zu dem Ergebnisse, dass mit der einfachen Heuristik 1/N die besseren Ergebnisse erzielt werden - etwas, was wohl auch Markowitz selbst erkannt hatte und anwendete. Man lernt daraus: Wenn man wenige Daten hat und die Voraussehbarkeit niedrig ist, funktionieren einfach Regeln besser als komplexe Strategien.

Im zweiten Beispiel ging es um ein Beispiel aus dem Einzelhandel, nämlich die Prognose, welche Kunden künftig weiter aktiv sind. Aus dem Kaufverhalten über einen längeren Zeitraum lässt sich anhand eines reichhaltigen Datensatzes für jeden Kunden nach dem Pareto/ NBD-Modell eine Prognose berechnen. Praktiker treffen ihre Entscheidung nach einer einfachen Distanz-Heuristik, in die lediglich die Zeitspanne seit dem letzten Einkauf eingeht: Wenn ein Kunde seit $m$ Monaten nicht gekauft hat, ist er inaktiv. Der Wert von m ist selbstverständlich von Branche zu Branche unterschiedlich, denn Güter des täglichen Bedarfs werden in kürzeren Abständen gekauft als Kleidung oder Einrichtungsgegenstände. Vergleichende Untersuchungen haben ergeben, dass die Distanz-Heuristik immer mindestens so gute Ergebnisse liefert, wie Pareto/NDB, oft bessere.

Das dritte Beispiel bezog sich auf die Preisbildung für Gebrauchtwagen. Hier hat man es gleichermaßen mit Unsicherheiten auf der Verkäufer- und auf der Käuferseite $\mathrm{zu}$ tun. Nicht selten hat man den Eindruck, dass zwei praktisch gleichwertige Fahrzeuge, im Fachjargon „Zwillinge“, zu deutlich unterschiedlichen Preisen angeboten werden. Woher kommt das? Herausgebildet hat sich bei den Gebrauchtwagenhändlern eine Treppenpreisheuristik. Danach senkt der Händler den ursprünglichen Angebotspreis immer nach dreißig Tagen um einen festen Wert, z. B. drei Prozent, bis ein Verkauf zustande kommt.

Als Resümee nannte Artinger drei verbreitete und kostspielige Irrtümer:

1. Die Zukunft ist ein verfluchtes Ärgernis nach dem anderen

2. Komplexe Probleme erfordern komplexe Lösungen

3. Mehr Information, Berechnung und Zeit ist immer besser

Die Erfahrungen zeigen, so Artinger, dass Experten immer dann schlecht mit ihrer Einschätzung abschneiden, wenn sich dramatische Änderungen vollziehen. In stabilen Verhältnissen, scheitern eher die Heuristiken, da ist meist der Experte überlegen.

In der Diskussion wurde deutlich, dass es im betrieblichen Alltag schwierig ist, dieses Plädoyer für Bauchentscheidungen umzusetzen. Es wird nur gelingen, wenn die Leitungsebene nicht defensives Entscheiden fördert, sondern für mutiges Entscheiden eintritt.

\section{Die Herkunft von Regeln hinterfragen}

Traditioneller Gast bei den Fachtagungen der SVP ist der Mathematiker und Philosoph Prof. Dr. Gunter Dueck, der jeweils auch an der Themenfindung mitwirkt. Sein Vor- 
tragstitel diesmal: „Risiko - Heuristik - Dogma: Wie man bei unvollständiger Information gute Entscheidungen trifft - und wie man es vermasseln kann!“ Anhand von Anekdoten aus seinem Berufsleben und Beobachtungen auf seinen vielen Reisen, etwa in unzweckmäßig gestalteten Hotelduschen, erklärte er, wie man immer wieder hinterfragen muss, wo bestehende Regeln und Heuristiken ihren Ursprung haben und ob ihre Voraussetzungen überhaupt noch Bestand haben. Bei den exorbitanten Preisen, die für manche Internet-Start-Ups bezahlt werden, wenn sie von einem der großen Internetkonzerne übernommen werden, lässt sich etwa erkennen, dass die Regel, man müsse sich die Umsatzzahlen angucken, bei Internet-Firmen nicht mehr zur Bewertung einer Firma gilt, sondern die Gewinnerwartung in der Zukunft den Kaufpreis bestimmt.

Nach wie vor gebe es jedoch Bereiche, wo man strikte Regeln aufstellen und befolgen muss, etwa wenn Infrastrukturen und Plattformen aufgebaut werden sollen und dafür Standards festgelegt werden müssen, dort, wo ein langsamer evolutionärer Wandel stattfindet oder wo lange Zeiten der Erfahrung vorliegen, die in Regeln gegossen werden können. Es sei keineswegs so, dass immer alles neu erfunden werden müsse.

Regeln nutzen im Bereich Kunst oder Spitzenforschung hingegen wenig oder gar nichts. Statistiken sind sinnlos in Zeiten des Wandels und beim Auftauchen disruptiver Technologien. Schützen sollte man sich auch davor, auf ,Idiotenregeln“ herein zu fallen. Eine weitere Gefahr bergen ,Korrelatalschäden', wenn aus Marktforschungs- oder anderen Umfragedaten extrahierte schlichte Korrelationen fälschlicherweise für Kausalitäten gehalten werden. Selbstironisch kokettierend nannte er sein neuestes Buch „schwarm dumm“, das auf Position 8 der Spiegel Bestseller-Liste steht. Daraus zu schließen, dass es gut sein müsse, wenn es auf der Liste steht, sei zwar eine falsche Heuristik, aber gut für ihn als Autor.

Wir lebten in einem Zeitalter, in dem sehr viele Berufe überflüssig werden, sodass sich jeder fragen müsse, biete ich als Person mehr als jemand, der zwei Stunden im Internet gesucht hat, bzw. was muss ich ändern, damit ich weiterhin gefragt bin.

Verschiedene Arten des Wandels erfordern unterschiedliche Menschen. Für Basis-Innovationen wird unternehmerisches Gespür benötigt, für Verbesserungen Ingenieure, die sich ein bestimmtes Problem herausgreifen, Effizienz wird durch gnadenlose Anwendung der BWL erreicht. Ideengeber sind oft genug nicht für die Umsetzung geeignet und brauchen entsprechende Partner. Markantes Beispiel sei die häufig wenig nachhaltige Forschungsförderung, bei der ein Forschungsthema geför- dert wird, ohne dass man sich vor der Bewilligung die Personen ganz genau ansieht, die das Thema bearbeiten sollen.

\section{Betriebliche Social Networks}

Nach der Mittagspause folgten vier Impulsreferate, in denen Profis erhellende Einblicke in ihre Berufspraxis gaben.

Die Market Intelligence-Spezialistin Alexandra Nelles hatte 2010 mit einer kleinen Gruppe von Kolleginnen und Kollegen bei Alcatel Lucent ein Social Network eingeführt, um Expertenwissen in der Firma auffindbar zu machen, eigenes Wissen in der Firma zu teilen und Ansprechpartner für eigene Fragestellungen schneller aufzufinden. Ihr Vortrag „Unternehmensinterne Barrieren aufbrechen - Wie man 74.000 Mitarbeiter als Quelle zur Wettbewerbsforschung nutzt!“ zeigte, wie es gelingen konnte, dass nach drei Jahre fast 74.000 Mitarbeiter das interne Netzwerk zum Wissensaustausch nutzten und um mit Kollegen über geographische und hierarchische Grenzen hinweg zu diskutieren.

Anfangs hatte jede Region, jede Unternehmenseinheit und jede Funktionseinheit eine eigen CI. Es gab sehr viele Informationen, die aber überall verteilt waren. Es entstand daher bei einer kleinen Gruppe von CI-Verantwortlichen die Idee, durch ein Competitive Intelligence Network (CIN) das verborgene Wissen der Firma zu nutzen.

Alcatel-Lucent ist einer der weltweit führenden Hersteller und Anbieter im Bereich Telekommunikationsund Netzwerkausrüstung. Von daher hatten die Beschäftigten eine natürliche Affinität zu Netzdiensten. Schlüssel zum Erfolg waren jedoch - wen wundert's - die beteiligten Personen. Eine kleine Gruppe von engagierten und aktiven CI-Spezialisten, die auch sich selbst die Arbeit erleichtern wollten, indem sie zur Unterstützung des Informationsaustauschs eine technische Netzinfrastruktur aufgebaut haben, und ein CIO, der die Initiative offensiv unterstützt hat. Kernanliegen war es, „die Silos einzureißen“, also die vielen lokalen Informationsbestände zugunsten einer konzernweiten Plattform aufzugeben. Fragen sollten nicht mehr bilateral gestellt und beantwortet werden, sondern betriebsöffentlich auf der Plattform. Auch hier wurde die Erfahrung gemacht, dass nicht die Werkzeuge, sondern die Verhaltensweisen Erfolg oder Misserfolg bestimmen. Und die Zusammenarbeit hat nicht nur die CI-Analysen unterstützt, sondern auch ihre Qualität verbessert. Als Fazit aus ihren Erfahrungen nannte sie die fünf Schritte zum Erfolg: 
1. Klare Ziele und Zielpersonen definieren, es geht darum Wissen über Wettbewerber zu sammeln

2. Schlüsselpositionen gut besetzen, einen executive sponsor, der das Projekt unterstützt, ein advocat, der sie Strategie vorantreibt, Moderatoren für die täglichen Aufgaben und die Schulung, Schlüsselpersonen in den einzelnen CI-Einheiten

3. Starten und Erfahrungen sammeln (Softlaunch), keine Bürokratie, sondern nur einen einfachen Code of Conduct aufstellen, auf Selbstregulierung setzen, einfache Bedienungsanleitungen und Kontaktdaten für Nachfragen zur Verfügung stellen

4. Fördern, führen, moderieren, dazu gehören auch Schulungen, etwa für eine gute Schlagwortvergabe, die das Finden von Informationen auf der Plattform erleichtert, oder mit Juristen darüber, was über Mitbewerber gesagt werden darf und was nicht

5. Beobachten, beharrlich sein und feiern, zwischendurch immer wieder motivieren, provokative Fragen als Stimulus stellen, Spielaktionen durchführen, Erfolge anerkennen und zum Jahrestag der Einführung richtig feiern

Auch der nächste Praxisvortag von Christian Hofer, Chief Analyst Officer bei SAP befasste sich mit dem Einsatz von „Social Media im Bereich Markt- und Wettbewerbsforschung!“

Christian Hofer gehört zur Pilotgruppe der SAP SE, die den internen Roll-out und sinnvolle Nutzungen von Social Media Tools für verschiedene Unternehmensbereiche erarbeitet. Daneben absolvierte er ein berufsbegleitendes Studium zum Competitive Intelligence Engineer (CIE) an der Graduate School Rhein-Neckar (Mannheim). Seine Erfahrungen deckten sich mit denen bei Alcatel Lucent. Zu den Anforderungen an die Werkzeuge gehört, dass sie auf allen stationären und mobilen Endgeräten funktionieren und einen social graph bieten. Es ist wichtig, dass die komplette Palette an Werkzeugen genutzt wird, Status Update, Fragen, Diskussionen, Umfragen, Blogs, Wikis, eine Plattform für Crowdsourcing. Alles, was extern verfügbar ist und genutzt wird, soll auch intern für die Markt- und Wettbewerbsanalyse eingesetzt werden können. Man muss sich darauf einstellen, dass die Einführung nicht von heute auf morgen klappt, Eine Zeitlang muss man mit gutem Beispiel vorangehen, relevante Inhalte, Meinungen und Perspektiven - keine Links - einstellen, irgendwann tritt dann der Netzwerkeffekt ein und die Gemeinschaft beginnt organisch zu wachsen.

\section{Aufklärung mit Google AdWords}

Dr. Christoph Gummersbach, mit seiner Firma Webfield Consulting selbstständiger Berater, Referent und Seminarleiter zu Google AdWords, Google Analytics und Suchmaschinenoptimierung, sprach über,,Pre-Sales Intelligence mit Google Analytics“ und präsentierte eine überraschend einfache und pragmatische Lösung zur Unterstützung des Außendienstes durch die Online-MarketingAbteilung.

Meist sind der Vertrieb und das Online Marketing in einem Unternehmen getrennte Welten. Im Bereich B2B liegen die Conversion Rates der Leads bei ca. einem Prozent. Doch wie lassen sich auch über die 99 Prozent Informationen gewinnen? Jeder Webbesucher hinterlässt bei jedem Besuch einer Website Spuren, nämlich die eigne IP-Adresse. Die meisten Firmen haben eigene IP-Bereiche, sodass sie sich identifizieren lassen, auch wenn bei der Anonymisierung das letzte Oktett der IP-Adresse gelöscht wird. Während einer Google-AdWords-Kampagne lässt sich dann auch das Suchinteresse feststellen. Beides zusammen ist eine wertvolle Information für den Vertrieb. Aus der Kombination von Land, Region, Stadt, Internetanbieter - also der Firma, um die es geht - Suchanfrage und Datum lassen sich valide Markstudien anfertigen. Allerdings sind diese sechs Dimensionsspalten mit Google Analytics nicht abbildbar. Es gibt jedoch eine Programmierschnittstelle mit der ein Google Analytics Zusatzprogramm für Google Tabelle geschrieben worden ist und mit dem eine siebenspaltige Tabelle erzeugt werden kann. Damit lassen sich Reports für die Vertriebsteams zu einzelnen Regionen oder Branchen anfertigen und für die Key Account Manager einzelner Unternehmen Dossiers über das aktuelle Suchverhalten der Zielgruppe zusammenstellen.

Schlüssel sind die eigenen AdWords, die man im Rahmen einer Kampagne quasi als Leimspur auslegt.

Tim Brouwer wies in der anschließenden Diskussion darauf hin, dass man als CI-Abteilung diese Möglichkeit, den Vertrieb zu unterstützen, für den ROI der eigenen Abteilung nutzen kann.

\section{Sanfte Aufklärung}

Einen wertvollen Blick in die tägliche Praxis der Informationsbeschaffung bot Michael Heimann von der Evonik Industries AG mit seinem Vortrag „Alternative Methoden der Competitive Intelligence - Was so nicht im BWLLehrbuch steht!“ bzw. „Was Sie schon immer über Ihren Wettbewerber wissen wollten, aber nie $\mathrm{zu}$ fragen wag- 
ten“. Er ist seit über 18 Jahren als Information Specialist der Evonik Industries AG in der Abteilung Information Intelligence tätig und verrichtet aus seiner Sicht eine investigative Arbeit. Mit einer Mischung aus kriminalistischer Intelligenz und Pragmatismus fügt er aus vielen Puzzleteilen kontinuierlich oder auftragsbezogene durch Beobachtung von Firmen, Branchen, Märkten, Forschungsaktivitäten und technologischen Entwicklungen ein Bild der Wettbewerber zusammen.

Das sollte jedoch nicht zufällig geschehen, vielmehr rät er dazu, einen Intelligence Collection Plan aufzustellen. Darin wird die Aufklärungsaufgabe in unterschiedliche Fragestellungen herunter gebrochen und dann $\mathrm{zu}$ jeder Frage die zugehörigen Indikatoren und die infrage kommenden Quellen aufgeführt. Messeauftritte sind für ihn „ein Schlaraffenland der Information“, weil dort erfahrungsgemäß das Standpersonal froh ist, wenn einmal jemand kommt, der sich für die Exponate interessiert. Aus solchen persönlichen Kontakten ergeben sich unbeabsichtigte Preisgaben von wichtigen Informationen.

Wichtige Informationsquellen für anstehende Betriebserweiterungen sind Stellenausschreibungen, Ausschreibungsdatenbanken und Web 2.0-Dienste. Um mehr über die Produktionsverfahren zu erfahren, eignen sich Patentanmeldungen oder Warenproben, die dann im eigenen Unternehmen untersucht werden können. Für Produkte, die nicht im Einzelhandel anonym gekauft werden können, bietet sich auch der Einsatz von Firmen wie SVP zum Mystery Shopping an, vorausgesetzt der Lieferant

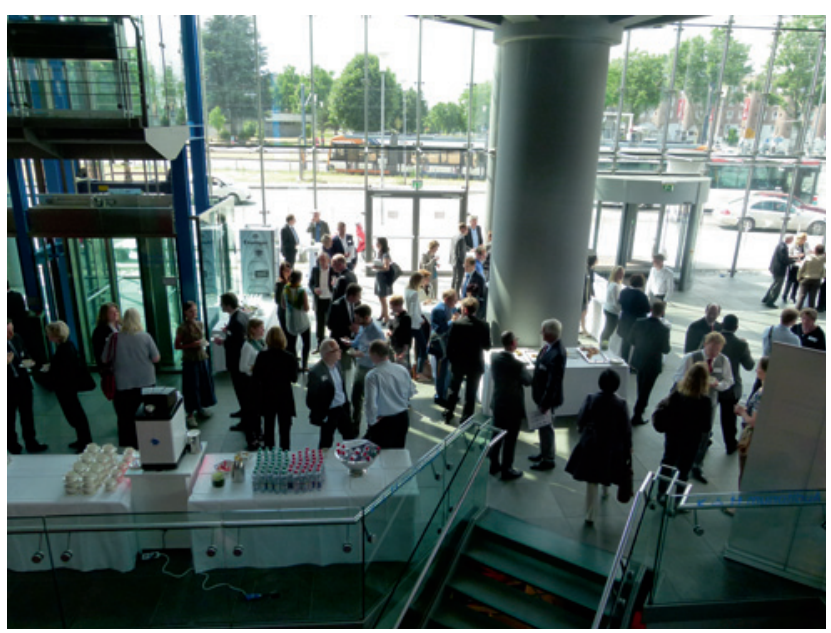

Abbildung 2: Transparenz bot nicht nur das Gebäude der Print \& Media Akademy, auch die Vorträge und Pausengespräche sorgten für neue Einsichten und Aha-Erlebnisse (Foto: SVP). verlangt keine Endverbleibserklärung. Eine wichtige Informationsquelle sind ferner Ein- und Ausfuhrlisten von Behörden wie den Zollämtern. Die Daten des Global Trade Tracker lassen sich mit www.begraphic.com visualisieren, sodass man die Handelsströme anschaulich darstellen und analysieren kann. Über die Standorte und die Bautätigkeit von Wettbewerbern, etwa den Bau neuer Anlagen, lässt sich auch durch die Auswertung von Satellitenaufnahmen oder Aufnahmen aus Google Earth etwas erfahren. In Deutschland ist es auch erlaubt Luftbildaufnahmen zu machen. Außendienstmitarbeiter können Fotos, die sie mit dem Smartphone an ihren Einsatzorten machen, inzwischen unmittelbar auf die heimische Plattform hochladen. Vor allem aber plädierte Heimann für die sanfte Aufklärungsmethode, die Nutzung von $\mathrm{Hu}$ man Intelligence anstelle von Computer Intelligence, das Networking in nationalen und internationalen Gremien und Gespräche.

\section{Nähkästchenplaudereien zum Schluss}

Den Abschluss der Fachtagung bildeten traditionell die moderierten Thementische, an denen sich interessierte Teilnehmerinnen und Teilnehmer zum Informations- und Erfahrungsaustausch zusammensetzten. Themen waren: Best in Class - Wettbewerbsbeobachtung, Market Intelligence - Software; Soziales Firmennetzwerk; Risiko und Unsicherheit sowie Trends und die praktischen Auswirkungen in vielen Branchen. Wie schon die Gespräche in den Pausen, war dieser Austausch sehr offen, kooperativ und produktiv und dauerte bis in den Abend.

Interessante Einblicke, offene Gespräche, beste Verpflegung, aufmerksame Veranstalter, professionelle Organisation, das freundliche Ambiente und sonniges Wetter machten die Fachtagung erneut zu einem anregenden Tag, von dem sicherlich jeder und jede etwas in den beruflichen Alltag mitnehmen konnte. Man darf sich schon aufs nächste Jahr freuen, dann findet am 14. Juni die nächste Fachtagung der SVP statt. Themenvorschläge nehmen die Veranstalter gerne entgegen. Die Vortragsfolien werden auf der Website www.market-intelligence. info zur Verfügung gestellt.

Deskriptoren: Tagung, Marktforschung, Market Intelligence, Risiko, Heuristik, Problemlösen, Informationsquelle, Soziales Netz 